\title{
Aplicação de método estatístico no estudo da influência do peróxido de hidrogênio e do borohidreto de sódio na síntese de nanoparticulas de prata (AGNPS)
}

\section{Application of statistical method in the study of the influence of hydrogen peroxide and sodium borohydride on silver nanoparticle synthesis (AGNPS)}

José Filipe Bacalhau Rodrigues ${ }^{1,4}$, Paula Emely de Souza Brandão ${ }^{2,4}$, Pedro Queiroz Guimarães ${ }^{2,4}$, Maria Roberta de Olibeira Pinto ${ }^{2,4}$, Renate Maria Ramos Wellen ${ }^{3}$, Marcus Vinicius Lia Fook ${ }^{1,4}$

\footnotetext{
${ }^{1}$ Universidade Federal de Campina Grande - UFCG, CEP: 58429-900, Campina Grande, PB, Brasil.

${ }^{2}$ Universidade Estadual da Paraíba - UEPB, CEP: 58429-500, Campina Grande, PB, Brasil.

${ }^{3}$ Universidade Federal da Paraíba - UFPB, CEP: 58297-000, João Pessoa, PB, Brasil.

${ }^{4}$ Laboratório de Avaliação e Desenvolvimento de Biomateriais do Nordeste - CertBio/UFCG, CEP: 58429-500, Campina Grande, PB, Brasil.

e-mail: filipe.rodrigues@certbio.ufcg.edu.br, viniciusliafook@yahoo.com.br, paulabrandaopb12@gmail.com, roberta1_oliveira@hotmail.com,pedro.queiroz@certbio.ufcg.edu.br
}

\section{RESUMO}

Nanomateriais vem atraindo bastante atenção nos últimos anos em virtude das suas propriedades que permitem aplicações em diversas áreas como: química, física, medicina, fármacos e engenharias. No universo dos nanomaterias, as nanopartículas de prata (AgNPs) tem despertado o interesse de vários pesquisadores devido ao seu potencial bactericida. Estudos recentes correlacionaram a eficácia da atividade antimicrobiana com a razão superfície-volume, morfologia, polidispersividade, tamanho de partícula e estabilidade das AgNPs. Este trabalho teve como objetivo compreender a influência do borohidreto de sódio $\left(\mathrm{NaBH}_{4}\right)$ e do peróxido de hidrogênio $\left(\mathrm{H}_{2} \mathrm{O}_{2}\right)$ nas propriedades das AgNPs. A síntese das AgNPs foi realizada através da redução química de íons de prata com $\mathrm{NaBH}_{4}$, na presença de $\mathrm{H}_{2} \mathrm{O}_{2}$ e Citrato de Sódio (SCT). Para determinar a influência causada pelo $\mathrm{H}_{2} \mathrm{O}_{2}$ e $\mathrm{NaBH}_{4}$, foram empregados diferentes variações de concentrações e volumes e ao resultado foi aplicado o Teste de Fisher para determinar a significância entre essas variáveis. As amostras foram caracterizadas por Espalhamento Dinâmico de Luz (DLS) e Potencial Zeta. Como resultado, o Teste de Fisher em conjunto com os valores obtidos de tamanho de partícula, estabilidade e polidispersividade determinaram variáveis estatisticamente iguais, ou seja, as variáveis que não influenciam estatisticamente na formação das partículas, bem como, a influência causada pela variação do $\mathrm{H}_{2} \mathrm{O}_{2}$ e do $\mathrm{NaBH}_{4}$ nas propriedades das AgNPs. Por fim, a variação da concentração de $\mathrm{NaBH}_{4}$ e volume de $\mathrm{H}_{2} \mathrm{O}_{2}$ causam efeitos sob o diâmetro, estabilidade e polidispersividade de AgNPs os quais foram estudados e aqui estão descritos.

Palavras-chave: Nanociência, nanopartículas de prata, Teste de Fisher.

\section{ABSTRACT}

Nanomaterials has attracted a lot of attention in recent years due to its properties that allow applications in several areas such as chemistry, physics, medicine, pharmaceuticals, and engineering. In the nanomaterial universe, silver nanoparticles (AgNPs) have awakened the interest of several researchers because of their bactericidal potential. Recent studies have correlated the efficacy of antimicrobial activity with the surfacevolume ratio, morphology, polydispersity, particle size and AgNPs stability. This work aimed to understand the influence of sodium borohydride $\left(\mathrm{NaBH}_{4}\right)$ and hydrogen peroxide $\left(\mathrm{H}_{2} \mathrm{O}_{2}\right)$ on the AgNPs properties. The synthesis of AgNPs was performed by chemical reduction of silver ions with sodium borohydride in the presence of hydrogen peroxide and sodium citrate. To determine the influence caused by $\mathrm{H}_{2} \mathrm{O}_{2}$ and $\mathrm{NaBH}_{4}$, different concentrations and volume variations were used and a Fisher's exact test was applied to determine the 
significance between variables. The samples were characterized by Dynamic Light Scattering (DLS) and Zeta Potential. Results determined that the Fisher's exact test along with the obtained results of variables of particle size, stability and polydispersity are statistically the same, that is, the variables do not influence statistically the formation of the particles, as well as the influence caused by $\mathrm{H}_{2} \mathrm{O}_{2}$ and $\mathrm{NaBH}_{4}$ variation on the AgNPs properties. In summary, variation of $\mathrm{NaBH}_{4}$ concentration and $\mathrm{H}_{2} \mathrm{O}_{2}$ volume causes effects under the diameter, stability and polydispersity of AgNPs which have been studied and described herein.

Keywords: Nanoscience, silver nanoparticles, Fisher's exact test.

\section{INTRODUÇÃO}

A nanotecnologia tem ganhado crescente interesse nos últimos anos devido ao aumento das possibilidades de aplicações nas áreas de construção civil [1,2], indústria de alimentos [3], dispositivos eletroeletrônicos [4] e na medicina, principalmente associada aos biomateriais [5-7]. Elevada área superficial e baixa citotoxicidade são características que tornam interessante o uso de materiais nanoparticulados. Na literatura, vários trabalhos relatam a síntese de nanopartículas metálicas, tais como: Au [8-10], Ag [11-13], Co [14, 15] e Cd [16, 17].

Nanoparticulas de prata (AgNPs) possuem propriedades físicas, químicas e biológicas únicas, dentre elas estão: propriedades ópticas, catalíticas, antimicrobianas, fungicidas e bactericidas [13, 18, 19]. Estudos apontam que a ação antimicrobiana das AgNPs acontece diretamente na estrutura do DNA dos microorganismos, os mesmos são menos propensos a desenvolver resistência ao tratamento quando submetidos ao contato com as nanoparticulas (NPs), requerendo diversas mutações para suportar este tipo de tratamento [20].

Trabalhos relatam que existe uma correlação entre a eficiência bactericida das AgNPs com sua estrutura cristalográfica, superfície-volume, tamanho de partícula, presença de estabilizantes e morfologia [21-25]. Pesquisadores informam que essas propriedades podem ser controladas através de condições de síntese, como concentração de reagentes e ligantes, temperatura e tempo, contudo, essa afirmação ainda está em questão devido a sensibilidade da nucleação e crescimento das NPs serem sensíveis a pequenas mudanças destas condições. [26] Adicionalmente, outras pesquisas se voltam para obtenção de NPs através de novos agentes redutores (verdes e biológicos) [7, 18] e sua incorporação em novos materiais, contudo, sem entendimento e controle das propriedades [27]. Assim, é de grande importância compreender a influência de cada fator na nucleação e crescimento das AgNPs.

$\mathrm{O}$ entendimento da influência dos reagentes a partir do uso de ferramentas estatísticas na síntese, no tamanho, morfologia e estabilidade de AgNPs se apresenta como potencial inovação para estudos que visam a aplicação desse tipo de NPs. Dessa forma, este trabalho teve como objetivo compreender a influência do borohidreto de sódio $\left(\mathrm{NaBH}_{4}\right)$ e do peróxido de hidrogênio $\left(\mathrm{H}_{2} \mathrm{O}_{2}\right)$ nas propriedades das AgNPs.

\section{MATERIAIS E MÉTODOS}

\subsection{Materiais}

Nitrato de prata $\left(\mathrm{AgNO}_{3}\right)$, Citrato de Sódio Tribásico Dihidratado $\left(\mathrm{Na}_{3} \mathrm{C}_{6} \mathrm{H}_{5} \mathrm{O}_{7} \cdot 2 \mathrm{H}_{2} \mathrm{O}\right)$ e Peróxido de Hidrogênio 35\% $\left(\mathrm{H}_{2} \mathrm{O}_{2}\right)$ foram adquiridos da Neon (São Paulo, Brasil). Borohidreto de Sódio $\left(\mathrm{NaBH}_{4}\right)$ foi adquirido na Sigma-Aldrich (Missouri, Estados Unidos). As soluções aquosas foram preparadas com água ultrapura, obtida a partir de um Sistema Master System MS2000 GEHAKA (São Paulo, Brasil).

\subsection{Métodos}

Na síntese de AgNPs foi utilizada uma modificação do método de redução química, descrito por ZHANG, LI [28]. Este método possibilita a obtenção de nanoprismas de prata de forma simples e rápida. Em um becker com $30 \mathrm{~mL}$ de água ultrapura foram adicionados $30 \mu \mathrm{L}$ de uma solução de $\operatorname{AgNO}_{3}\left(0,1\right.$ mmol.L $\left.{ }^{-1}\right), 1,5 \mathrm{~mL}$ de Citrato de Sódio (SCT)(90 mmol.L $\left.{ }^{-1}\right), \mathrm{H}_{2} \mathrm{O}_{2}$ (35\% P.A.) e $200 \mu \mathrm{L}$ de $\mathrm{NaBH}_{4}$. Em seguida, a solução foi mantida sob agitação vigorosa por $\sim 3$ minutos à temperatura ambiente. Verificou-se uma mudança da coloração da solução que passou de translúcida para amarela e, subsequentemente, azul.

Um total de 12 amostras foram sintetizadas variando concentrações e volumes de $\mathrm{H}_{2} \mathrm{O}_{2}$ e $\mathrm{NaBH}_{4}$ de acordo com a Tabela 1: 
Tabela 1: Variações de concentração e volume de $\mathrm{H}_{2} \mathrm{O}_{2}$ e $\mathrm{NaBH}_{4}$ utilizados na síntese.

\begin{tabular}{c|c|c}
\hline VARIÁVEL & AMOSTRA & VARIAÇÃO \\
\hline \multirow{4}{*}{$\mathrm{H}_{2} \mathrm{O}_{2}($ Volume - $\mu \mathrm{L})$} & $\mathrm{H} 1$ & 30 \\
\cline { 2 - 3 } & $\mathrm{H} 2$ & 60 \\
\cline { 2 - 3 } & $\mathrm{H} 3$ & 90 \\
\cline { 2 - 3 } & $\mathrm{H} 4$ & 100 \\
\cline { 2 - 3 } & $\mathrm{H} 5$ & 120 \\
\cline { 2 - 3 } & $\mathrm{H} 6$ & 150 \\
\hline \multirow{4}{*}{$\mathrm{NaBH}_{4}\left(\right.$ Concentração - mmol.L $\left.^{-1}\right)$} & $\mathrm{B} 1$ & 50 \\
\cline { 2 - 3 } & $\mathrm{B} 2$ & 60 \\
\cline { 2 - 3 } & $\mathrm{B} 3$ & 70 \\
\cline { 2 - 3 } & $\mathrm{B} 4$ & 80 \\
\cline { 2 - 3 } & $\mathrm{B} 5$ & 90 \\
\cline { 2 - 3 } & $\mathrm{B} 6$ & 100 \\
\hline
\end{tabular}

\subsection{Caracterização das AgNPs}

O DLS foi usado para determinação do tamanho e dispersão das AgNPs. As análises foram realizadas em um Instrumento ZetaPals da Brookhaven. As medidas foram conduzidas a temperatura de $25^{\circ} \mathrm{C}$ com um ângulo de espalhamento fixado em $90^{\circ}$.

A técnica de Potencial Zeta foi empregada para avaliar a estabilidade das AgNPs diante das diferentes condições de síntese. O software Action Stat foi utilizado na aplicação do Teste de Fisher.

\section{RESULTADOS E DISCUSSÃO}

$\mathrm{O} \mathrm{NaBH}_{4}$ possui função redutora e função secundária como agente de encapsulamento, podendo estabilizar AgNPs de acordo com o tempo de crescimento prolongado diante de altas concentrações. $\mathrm{O} \mathrm{H}_{2} \mathrm{O}_{2}$, já é bem conhecido em seu uso como agente oxidante [29], promovendo a formação de estruturas anisotrópicas. ZHANG, LI [28] em seus estudos relataram que, inicialmente, com a adição de $\mathrm{NaBH}_{4}$, os íons de prata são parcialmente reduzidos para formar AgNPs, que são temporariamente estabilizadas. Simultaneamente, o crescimento de NPs é inibido devido ao $\mathrm{H}_{2} \mathrm{O}_{2}$. Como resultado do equilíbrio dinâmico entre a redução e a oxidação, a prata permanece na forma de nanopartículas de cor amarela clara. Com o consumo do $\mathrm{NaBH}_{4}$ durante a reação, a proteção dos íons borohidreto é enfraquecida, permitindo a produção de núcleos de prata.

A Figura 1 ilustra a evolução do diâmetro de NPs de acordo com a variação do $\mathrm{H}_{2} \mathrm{O}_{2}$ e $\mathrm{NaBH}_{4}$. Uma linha de tendência foi utilizada para melhor visualização dos resultados. 

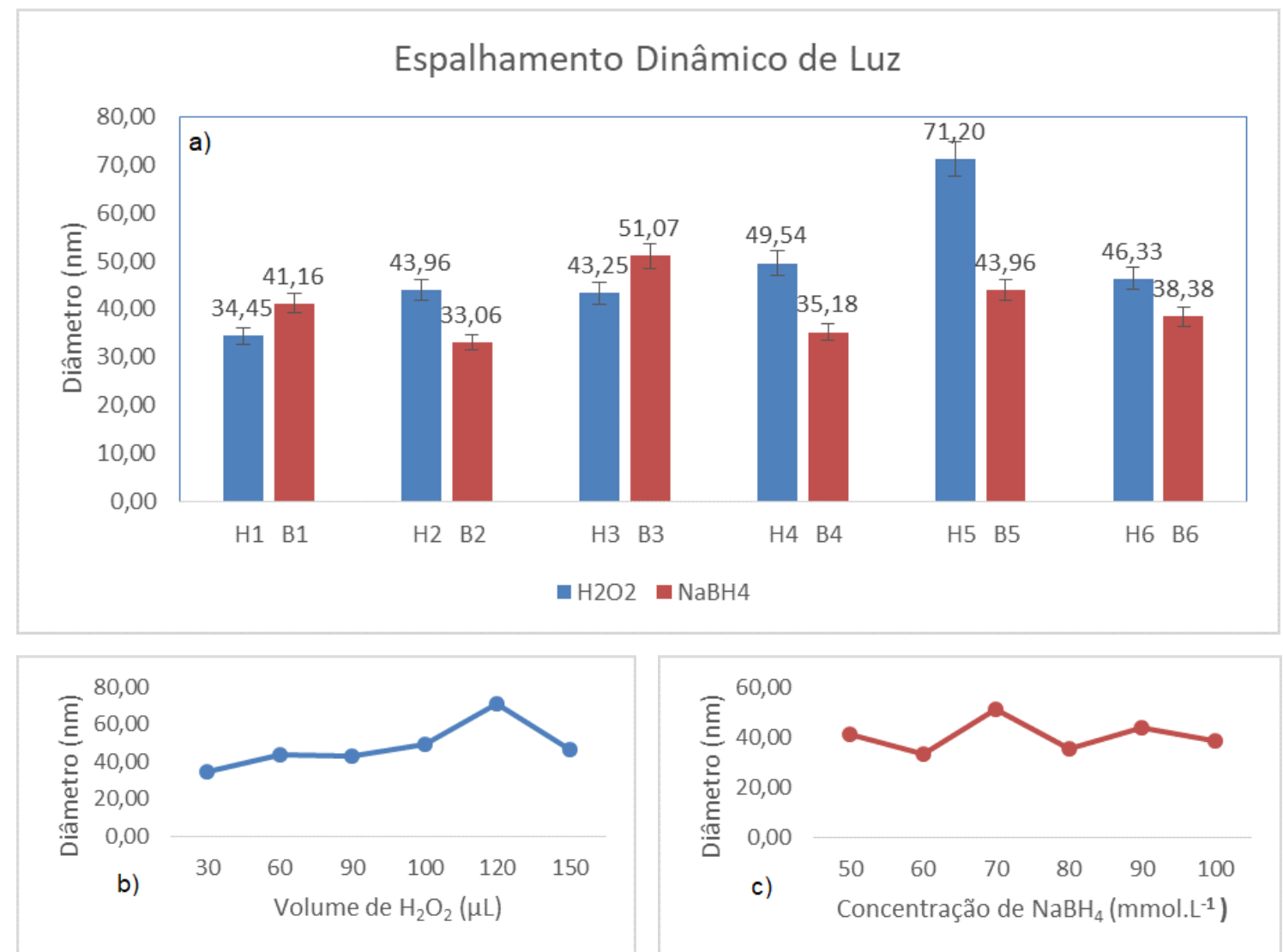

Figura 1: a) Comportamento do diâmetro hidrodinâmico das AgNPs em função da variação de $\mathrm{NaBH}_{4} \mathrm{e}_{2} \mathrm{O}_{2}$. b) Linha de tendência do diâmetro hidrodinâmico em função do $\mathrm{H}_{2} \mathrm{O}_{2}$. c) Linha de tendência do diâmetro hidrodinâmico em função do $\mathrm{NaBH}_{4}$.

De acordo com os resultados ilustrados na Figura $1 \mathrm{~b}$ ), observa-se que o aumento de $\mathrm{H}_{2} \mathrm{O}_{2}$ até o volume de $120 \mu \mathrm{L}$ proporcionou um aumento do diâmetro das NPs, possivelmente devido a sua ação no crescimento das NPs como também diminuiu o efeito retardante de crescimento gerado pelo $\mathrm{NaBH}_{4}$ devido a não existência de um equilíbrio dinâmico entre a redução dos íons de Ag e a dissolução oxidativa da prata metálica [28].

ZHANG, LI [28] relataram em seus estudos que o aumento da concentração de $\mathrm{NaBH}_{4}$ proporcionou uma redução da espessura das AgNPs. Nos resultados apresentados nesse trabalho o aumento da concentração de $\mathrm{NaBH}_{4}$ pouco contribuiu com a alteração do diâmetro das AgNPs visto que, o aumento da concentração de $\mathrm{NaBH}_{4}$ conduziu a uma produção mais lenta de AgNPs, reduzindo o tempo de inicialização de formação dos núcleos de NPs, no entanto, após passado seu efeito o crescimento do diâmetro das AgNPs foi pouco afetado.

A correlação entre a variação do diâmetro de partículas e da polidispersividade é ilustrado na Figura 2. Nota-se que existe uma tendência no comportamento do diâmetro versus polidispersividade diante da variação do $\mathrm{NaBH}_{4}$ e $\mathrm{H}_{2} \mathrm{O}_{2}$.

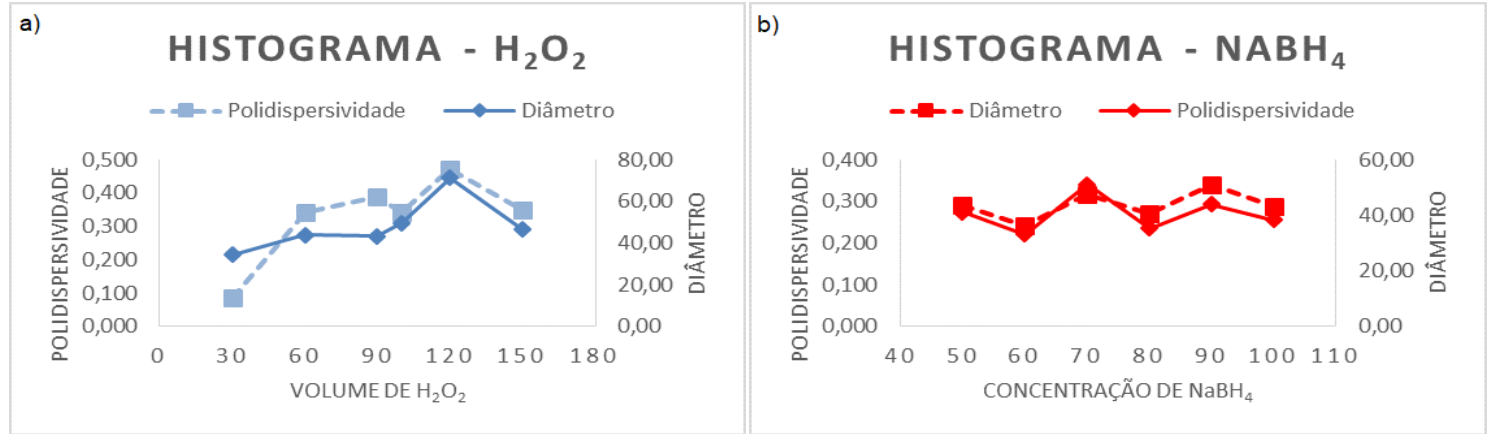

Figura 2: Polidispersividade versus Diâmetro. a) $\mathrm{H}_{2} \mathrm{O}_{2}$ e b) $\mathrm{NaBH}_{4}$. 
Pesquisas relatam a forma de crescimento de AgNPs semeadas e nucleadas, obtendo nanoparticulas com espessuras de 4nm para AgNPs ainda em fase de crescimento (sementes) e de até 10nm para AgNPs já nucleadas, contudo não correlacionaram a influência dos reagentes com a polidispersividade das AgNPs [26, 28, 30-32].

Analogamente aos efeitos causados no diâmetro de partícula, o aumento de $\mathrm{H}_{2} \mathrm{O}_{2}$ e $\mathrm{NaBH}_{4}$ causou aumento na distribuição dos tamanhos, favorecendo a formação de uma solução polidispersa, fora da idealidade >0,300 [33] (fator considerado negativo para NPs, visto que a obtenção de NPs monodispersas permite a correlação direta das propriedades das NPs do sistema obtido com as propriedades das NPs individuais) [34-36]. Dessa forma, quanto maior a monodispersividade, maior a homogeneidade das propriedades da amostra obtida.

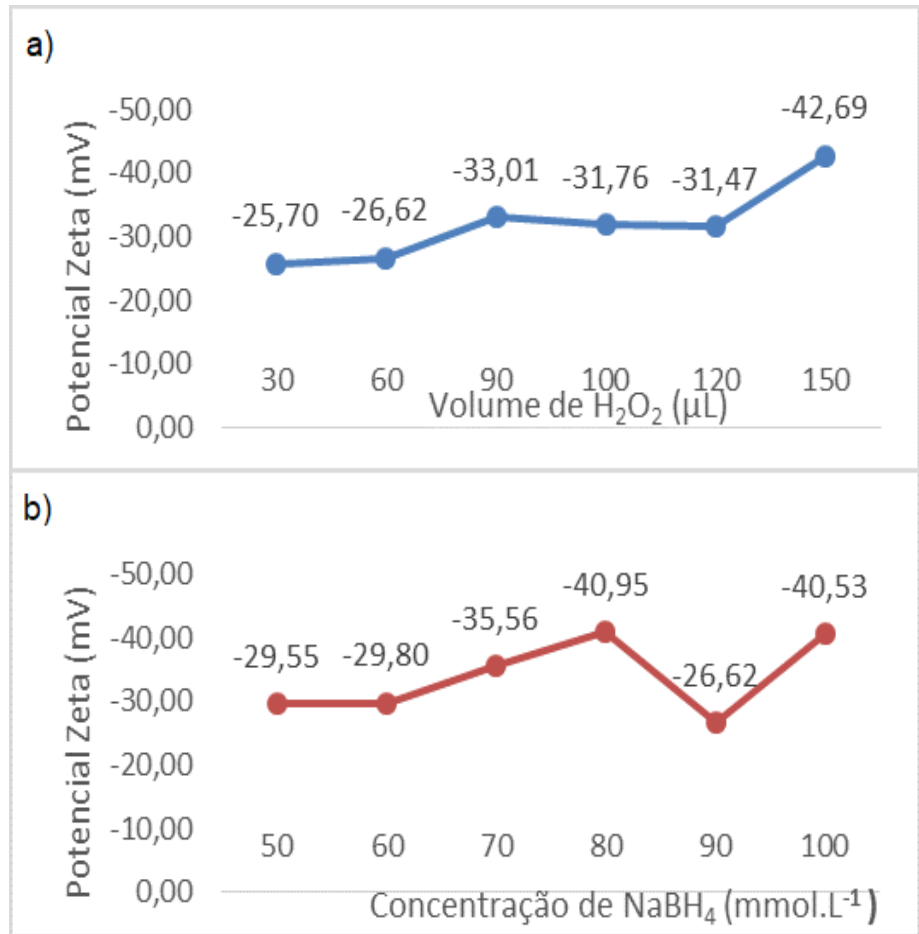

Figura 3: Comportamento da polidispersividade diante das variações de: a) $\mathrm{H}_{2} \mathrm{O}_{2}$ e b) $\mathrm{NaBH}_{4}$.

O potencial zeta é uma técnica que permite avaliar a estabilidade de dispersões coloidais através da medição do seu potencial eletro cinético[37]. A Figura 3 ilustra o comportamento da estabilidade das NPs em função do $\mathrm{H}_{2} \mathrm{O}_{2}$ e $\mathrm{NaBH}_{4}$.

Para todas as variações de concentração do $\mathrm{NaBH}_{4}$ e volume do $\mathrm{H}_{2} \mathrm{O}_{2}$ os resultados demonstram que a superfície das partículas estão carregadas negativamente. Valores de potencial zeta abaixo de $<-0,30 \mathrm{mV}$ é sinal de NPs estáveis devido a repulsão causada entre as NPs, impedindo sua aglomeração[38]. O aumento do volume de $\mathrm{H}_{2} \mathrm{O}_{2}$ favoreceu a estabilidade das partículas, demonstrando um aumento progressivo, fator este já esperado diante da atuação do $\mathrm{H}_{2} \mathrm{O}_{2}$ na remoção das partículas menos instáveis. $\mathrm{O}$ aumento das concentrações de $\mathrm{NaBH}_{4}$, analogamente ao $\mathrm{H}_{2} \mathrm{O}_{2}$ proporcionou uma melhoria na estabilidade das NPs. Isso se explica através de um possível efeito estabilizador sinérgico entre íons de citrato e íons de borohidreto, onde uma concentração maior de íons de borohidreto aumenta a ligação preferencial do citrato à faceta (111), levando à formação de nanopartículas mais estáveis [28].

O método de Fisher é utilizado para comparar todos os pares de médias controlando a taxa de erro ao nível de significância $\alpha$ para cada comparação. Uma vez que o nível de significância entre as médias é menor que 5\% rejeitamos a hipótese de igualdade. Valores localizados acima do nível de significância (5\%) são considerados estatisticamente iguais, ou seja, estatisticamente o efeito causado pelas variáveis comparadas nas propriedades físico-químicas é similar, não influenciando na formação de NPs. Por outro lado, valores abaixo do nível de significância são considerados estatisticamente diferentes (contribuem na formação de NPs). Em nosso estudo, o Teste de Fisher foi empregado para avaliar se existem diferenças significativas entre as médias obtidas para o diâmetro, polidispersividade e estabilidade. 
A Figura 4 ilustra o intervalo de confiança do Teste de Fisher em função da comparação das médias dos valores de diâmetro, polidispervisidade e potencial zeta.

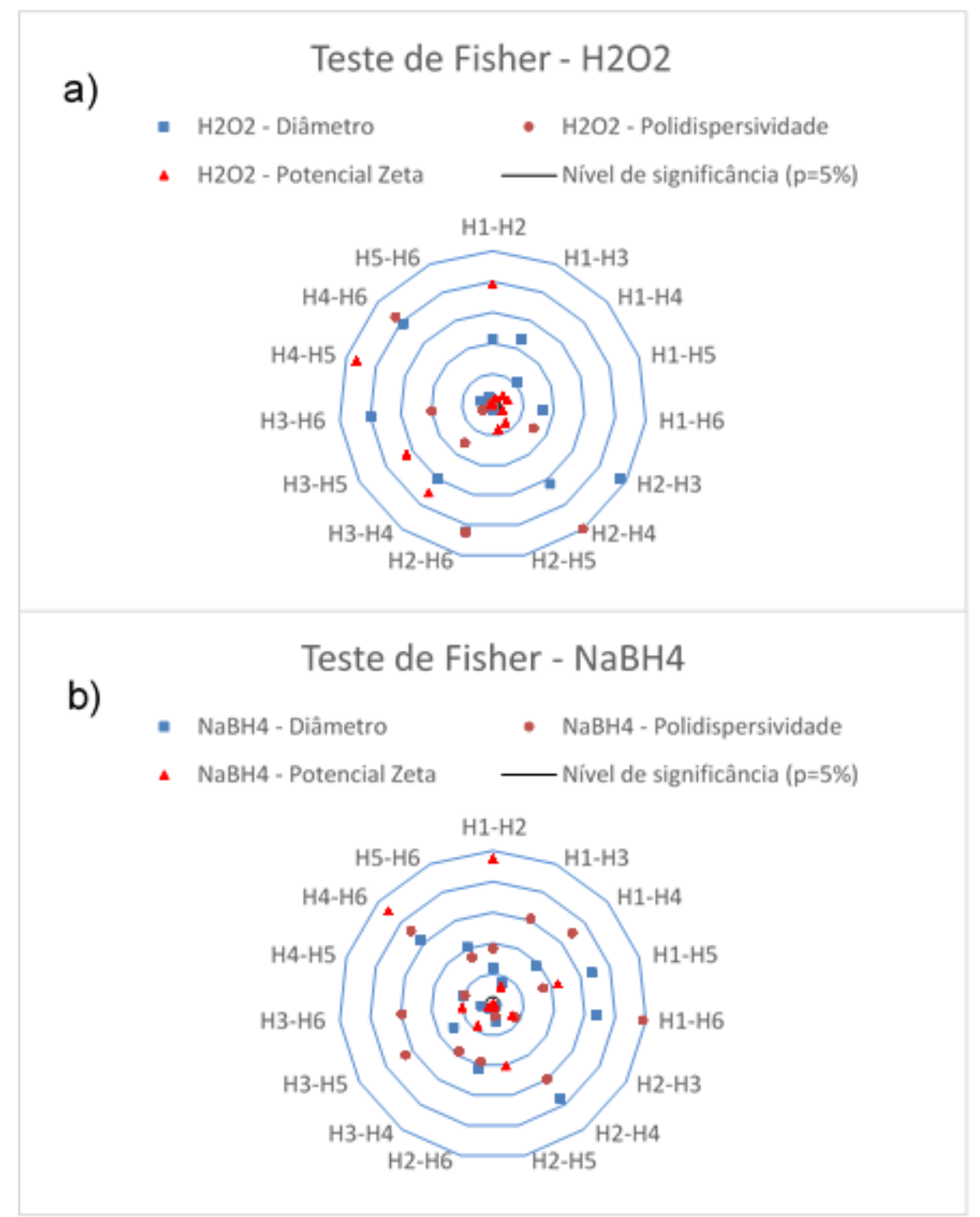

Figura 4: Intervalo de confiança do Teste de Fisher relativo a diferença das médias de diâmetro, polidispersividade e potencial zeta para: a) volume de $\mathrm{H}_{2} \mathrm{O}_{2}$ e b) concentração de $\mathrm{NaBH}_{4}$ em função dos valores da estatística do Teste de Fisher.

Observando os valores na Figura 4 a): diâmetro - todas as combinações $\mathrm{H}_{\mathrm{n}}-\mathrm{H}_{\mathrm{n}}$ com exceção das interações $\mathrm{H}_{1}-\mathrm{H}_{5}, \mathrm{H}_{2}-\mathrm{H}_{5}$ e $\mathrm{H}_{3}-\mathrm{H}_{5}$ (valores médios de diâmetro significativamente diferentes) estão acima do intervalo de confiança, contribuindo no crescimento das NPs (baixa influência no diâmetro de partícula); com relação ao potencial zeta e polidispersividade, todas as médias comparadas com a variação $\mathrm{H}_{6}(150 \mu \mathrm{L})$ e $\mathrm{H}_{1}$ $(30 \mu \mathrm{L})$, respectivamente, demonstraram-se estatisticamente diferentes $(\mathrm{p}<5 \%)$ demonstrando a grande influência do $\mathrm{H}_{2} \mathrm{O}_{2}$ nestas propriedades.

$\mathrm{Na}$ Figura 4 b): diâmetro - valores do teste Fisher em comparação das médias $\mathrm{H}_{2}-\mathrm{H}_{3}$ e $\mathrm{H}_{3}-\mathrm{H}_{4}$ demonstraram-se estatisticamente diferentes, contrário ao observado na avaliação dos resultados químicos onde a variação da concentração de $\mathrm{NaBH}_{4}$ pouco contribui para o aumento e/ou variação do diâmetro de NPs; potencial zeta - variações na concentração de $\mathrm{NaBH}_{4}$ na faixa de $50-100 \mathrm{mmol}^{-1} \mathrm{~L}^{-1}$ são estatisticamente iguais ( $>$ >5\%); Polidispersividade - diferenças significativas quando comparadas as variáveis grandes variações de concentração $\left(\mathrm{H}_{4}, \mathrm{H}_{5}\right.$ e $\mathrm{H}_{6}$ versus $\mathrm{H}_{1}, \mathrm{H}_{2}$ e $\left.\mathrm{H}_{3}\right)$. 


\section{CONCLUSÕES}

Os resultados demonstraram que o aumento do volume de $\mathrm{H}_{2} \mathrm{O}_{2}$ até o volume de $120 \mu \mathrm{L}$ proporcionou o aumento do diâmetro das NPs, removendo partículas instáveis durante o processo de crescimento. O comportamento da polidispersividade foi similar ao observado no diâmetro quando estudadas as variações de $\mathrm{H}_{2} \mathrm{O}_{2} \mathrm{e}$ $\mathrm{NaBH}_{4}$. Adicionalmente, maiores concentrações de $\mathrm{NaBH}_{4}$ e volumes de $\mathrm{H}_{2} \mathrm{O}_{2}$ proporcionaram aumento na estabilidade das NPs. No Teste de Fisher todas as concentrações de $\mathrm{NaBH}_{4}$ não influenciaram estatisticamente os valores de polidispersividade. Por outro lado, a estabilidade das AgNPs foi estatisticamente diferente para volumes mais altos de $\mathrm{H}_{2} \mathrm{O}_{2}$, confirmando a forte influência do $\mathrm{H}_{2} \mathrm{O}_{2}$ na estabilidade de NPs. Os resultados deste trabalho possibilitaram a compreensão do efeito causado pela variação da concentração de $\mathrm{NaBH}_{4} \mathrm{e}$ volume de $\mathrm{H}_{2} \mathrm{O}_{2}$ sob o diâmetro, estabilidade e polidispersividade de AgNPs por meio do uso de técnicas de caracterização e ferramentas estatísticas.

\section{AGRADECIMENTOS}

A Coordenação de Melhoria do Ensino Superior Pessoal (CAPES), ao Conselho Nacional de Desenvolvimento Científico e Tecnológico (CNPq), ao Laboratório de Avaliação e Desenvolvimento de Biomateriais do Nordeste (CERTBIO) e a Universidade Federal de Campina Grande (UFCG).

\section{BIBLIOGRAFIA}

[1] GAUVIN, F., ROBERT, M., "Durability study of vinylester/silicate nanocomposites for civil engineering applications", Polymer Degradation and Stability, v. 121, pp. 359-68, 2015.

[2] PAYAKANITI, P., PINITSOONTHORN, S., THONGBAI, P., AMORNKITBAMRUNG, V., CHINDAPRASIRT, P., "Effects of carbon fiber on mechanical and electrical properties of fly ash geopolymer composite", Materials Today: Proceedings, v. 5, n. 6, pp. 14017-25, 2018.

[3] HE, X., HWANG, H.-M., "Nanotechnology in food science: functionality, applicability, and safety assessment", journal of food and drug analysis, v. 24, n. 4, pp. 671-81, 2016.

[4] RAMIREZ-LEE, M. A., AGUIRRE-BAÑUELOS, P., MARTINEZ-CUEVAS, P. P., ESPINOSATANGUMA, R., CHI-AHUMADA, E., MARTINEZ-CASTAÑON, G. A., GONZALEZ, C., "Evaluation of cardiovascular responses to silver nanoparticles (AgNPs) in spontaneously hypertensive rats", Nanomedicine: Nanotechnology, Biology and Medicine, v. 14, n. 2, pp. 385-95, 2018.

[5] HASSANZADEH, P., ATYABI, F., DINARVAND, R., "Application of modelling and nanotechnologybased approaches: The emergence of breakthroughs in theranostics of central nervous system disorders", Life sciences, v. 182, pp. 93-103, 2017.

[6] YE, H., CHENG, J., YU, K., "In situ reduction of silver nanoparticles by gelatin to obtain porous silver nanoparticle/chitosan composites with enhanced antimicrobial and wound-healing activity", International journal of biological macromolecules, v. 121, pp. 633-42, 2019.

[7] GHIUȚĂ, I., CRISTEA, D., CROITORU, C., KOST, J., WENKERT, R., VYRIDES, I., ANAYIOTOS, A., MUNTEANU, D., "Characterization and antimicrobial activity of silver nanoparticles, biosynthesized using Bacillus species", Applied Surface Science, v. 438, pp. 66-73, 2018.

[8] IRFAN, M., MONIRUZZAMAN, M., AHMAD, T., MANDAL, P. C., BHATTACHARJEE, S., ABDULLAH, B., "Ionic liquid based extraction of flavonoids from Elaeis guineensis leaves and their applications for gold nanoparticles synthesis", Journal of Molecular Liquids, v. 241, pp. 270-8, 2017.

[9] ZULINA, N. A., BARANOV, M. A., KNIAZEV, K. I., KALIABIN, V. O., DENISYUK, I. Y., ACHOR, S. U., SITNIKOVA, V. E., "Nonlinear absorption enhancement of AuNPs based polymer nanocomposites", Optics \& Laser Technology, v. 103, pp. 396-400, 2018.

[10] LEE, H., LEE, D. G., "Gold nanoparticles induce a reactive oxygen species-independent apoptotic pathway in Escherichia coli", Colloids and Surfaces B: Biointerfaces, v. 167, pp. 1-7, 2018.

[11] LEE, S. J., HEO, M., LEE, D., HAN, S., MOON, J.-H., LIM, H.-N., KWON, I. K., "Preparation and characterization of antibacterial orthodontic resin containing silver nanoparticles", Applied Surface Science, v. 432, pp. 317-23, 2018.

[12] Petica, A., GAVRiliU, S., Lungu, M., BuRUnTEA, N., PANZARU, C., "Colloidal silver solutions with antimicrobial properties", Materials Science and Engineering: B, v. 152, n. 1-3, pp. 22-7, 2008. 
[13] PAOSEN, S., SAISING, J., WIRA SEPTAMA, A., PIYAWAN VORAVUTHIKUNCHAI, S., "Green synthesis of silver nanoparticles using plants from Myrtaceae family and characterization of their antibacterial activity", Materials Letters, v. 209, pp. 201-6, 2017.

[14] JAGADEESH, R. V., MURUGESAN, K., ALSHAMMARI, A. S., NEUMANN, H., POHL, M.-M., RADNIK, J., BELLER, M., "MOF-derived cobalt nanoparticles catalyze a general synthesis of amines", Science, v. 358, n. 6361, pp. 326-32, 2017.

[15] LIU, J., WANG, Z., YAN, X., JIAN, P., "Metallic cobalt nanoparticles imbedded into ordered mesoporous carbon: A non-precious metal catalyst with excellent hydrogenation performance", Journal of colloid and interface science, v. 505, pp. 789-95, 2017.

[16] HONG, L., CHEUNG, T.-L., RAO, N., OUYANG, Q., WANG, Y., ZENG, S., YANG, C., CUONG, D., CHONG, P. H. J., LIU, L., "Millifluidic synthesis of cadmium sulfide nanoparticles and their application in bioimaging", RSC Advances, v. 7, n. 58, pp. 36819-32, 2017.

[17] ZAHERA, M., KHAN, S. A., KHAN, I. A., ELGORBAN, A. M., BAHKALI, A. H., ALGHAMDI, S. M., KHAN, M. S., "Enhancing using glucose encapsulation, the efficacy of CdO NPs against multi-drug resistant Escherichia coli", Microbial pathogenesis, v. 119, pp. 42-8, 2018.

[18] HE, H., TAO, G., WANG, Y., CAI, R., GUO, P., CHEN, L., ZUO, H., ZHAO, P., XIA, Q., "In situ green synthesis and characterization of sericin-silver nanoparticle composite with effective antibacterial activity and good biocompatibility", Mater Sci Eng C Mater Biol Appl, v. 80, pp. 509-16, Nov 12017.

[19] LIU, G., HAIQI, G., LI, K., XIANG, J., LAN, T., ZHANG, Z., "Fabrication of silver nanoparticle sponge leather with durable antibacterial property", Journal of Colloid and Interface Science, v. 514, pp. 338-48, 2018.

[20] PAL, S., TAK, Y. K., SONG, J. M., "Does the antibacterial activity of silver nanoparticles depend on the shape of the nanoparticle? A study of the gram-negative bacterium Escherichia coli", Applied and environmental microbiology, v. 73, n. 6, pp. 1712-20, 2007.

[21] BAKER, C., PRADHAN, A., PAKSTIS, L., POCHAN, D. J., SHAH, S. I., "Synthesis and antibacterial properties of silver nanoparticles", Journal of nanoscience and nanotechnology, v. 5, n. 2, pp. 244-9, 2005.

[22] HAYNES, C. L., VAN DUYNE, R. P., "Plasmon-sampled surface-enhanced Raman excitation spectroscopy", The Journal of Physical Chemistry B, v. 107, n. 30, pp. 7426-33, 2003.

[23] JIN, R., CAO, Y., MIRKIN, C. A., KELLY, K., SCHATZ, G. C., ZHENG, J., "Photoinduced conversion of silver nanospheres to nanoprisms", science, v. 294, n. 5548, pp. 1901-3, 2001.

[24] PASTORIZA-SANTOS, I., LIZ-MARZÁN, L. M., "Synthesis of silver nanoprisms in DMF", Nano letters, v. 2, n. 8, pp. 903-5, 2002.

[25] CHEN, S., CARROLL, D. L., "Synthesis and characterization of truncated triangular silver nanoplates", Nano letters, v. 2, n. 9, pp. 1003-7, 2002.

[26] ZHANG, Q., HU, Y., GUO, S., GOEBL, J., YIN, Y., "Seeded growth of uniform Ag nanoplates with high aspect ratio and widely tunable surface plasmon bands", Nano letters, v. 10, n. 12, pp. 5037-42, 2010.

[27] GABRIEL, J. S., GONZAGA, V. A. M., POLI, A. L., SCHMITT, C. C., "Photochemical synthesis of silver nanoparticles on chitosans/montmorillonite nanocomposite films and antibacterial activity", Carbohydrate Polymers, v. 171, pp. 202-10, 2017.

[28] ZHANG, Q., LI, N., GOEBL, J., LU, Z., YIN, Y., "A systematic study of the synthesis of silver nanoplates: is citrate a "magic" reagent?", J Am Chem Soc, v. 133, n. 46, pp. 18931-9, Nov 232011.

[29] MULVIHILL, M. J., LING, X. Y., HENZIE, J., YANG, P., "Anisotropic etching of silver nanoparticles for plasmonic structures capable of single-particle SERS", Journal of the American Chemical Society, v. 132, n. 1, pp. 268-74, 2009.

[30] GOEBL, J., ZHANG, Q., HE, L., YIN, Y., "Monitoring the shape evolution of silver nanoplates: a marker study", Angewandte Chemie International Edition, v. 51, n. 2, pp. 552-5, 2012.

[31] SUN, Y., XIA, Y., "Shape-controlled synthesis of gold and silver nanoparticles", Science, v. 298, n. 5601, pp. 2176-9, 2002.

[32] LI, N., ZHANG, Q., QUINLIVAN, S., GOEBL, J., GAN, Y., YIN, Y., "H2O2 - Aided Seed - Mediated Synthesis of Silver Nanoplates with Improved Yield and Efficiency", ChemPhysChem, v. 13, n. 10, pp. 252630, 2012. 
[33] MAHL, D., DIENDORF, J., MEYER-ZAIKA, W., EPPLE, M., "Possibilities and limitations of different analytical methods for the size determination of a bimodal dispersion of metallic nanoparticles", Colloids and Surfaces A: Physicochemical and Engineering Aspects, v. 377, n. 1-3, pp. 386-92, 2011.

[34] SUGIMOTO, T., "Preparation of monodispersed colloidal particles", Advances in Colloid and Interface Science, v. 28, pp. 65-108, 1987.

[35] MARTINEZ-CASTANON, G., NINO-MARTINEZ, N., MARTINEZ-GUTIERREZ, F., MARTINEZMENDOZA, J., RUIZ, F., "Synthesis and antibacterial activity of silver nanoparticles with different sizes", Journal of Nanoparticle Research, v. 10, n. 8, pp. 1343-8, 2008.

[36] KUMAR, B., SMITA, K., CUMBAL, L., DEBUT, A., "Green synthesis of silver nanoparticles using Andean blackberry fruit extract", Saudi journal of biological sciences, v. 24, n. 1, pp. 45-50, 2017.

[37] EL BADAWY, A. M., SILVA, R. G., MORRIS, B., SCHECKEL, K. G., SUIDAN, M. T., TOLAYMAT, T. M., "Surface charge-dependent toxicity of silver nanoparticles", Environmental science \& technology, v. 45, n. 1, pp. 283-7, 2010.

[38] DOANE, T. L., CHUANG, C.-H., HILL, R. J., BURDA, C., "Nanoparticle $\zeta$-Potentials", Accounts of Chemical Research, v. 45, n. 3, pp. 317-26, 2012/03/20 2012.

\section{ORCID}

José Filipe Bacalhau Rodrigues Renate Maria Ramos Wellen Paula Emely de Souza Brandão Marcus Vinicius Lia Fook Maria Roberta de Oliveira Pinto Pedro Queiroz Guimarães https://orcid.org/0000-0002-3916-6214

https://orcid.org/0000-0002-3565-7366

https://orcid.org/0000-0001-7967-5243

https://orcid.org/0000-0002-8566-920X

https://orcid.org/0000-0002-2151-3564

https://orcid.org/0000-0003-0585-5756 